\title{
Effect of radial magnetic field on the natural convection in a semi- circular curved enclosure for different aspect ratios
}

\author{
NARENDRA LAXMAN GAJBHIYE ${ }^{1}$ and VINAYAK ESWARAN ${ }^{2, *}$ \\ ${ }^{1}$ Department of Mechanical Engineering, Maulana Azad National Institute of Technology Bhopal, \\ Bhopal 462003, India \\ ${ }^{2}$ Department of Mechanical and Aerospace Engineering, Indian Institute of Technology Hyderabad, \\ Hyderabad 502285, India \\ e-mail: nlgajbhiye@gmail.com; eswar@iith.ac.in
}

MS received 8 June 2018; revised 21 November 2018; accepted 30 November 2018; published online 14 March 2019

\begin{abstract}
The problem of natural convection in a (2D) semi-circular curved enclosure in the presence of a radial magnetic field is numerically studied in this paper. The selected configuration is such that the convective flow is driven by a mean temperature gradient also directed radially, and the effects of enclosure aspect ratio and the strength of the applied magnetic field are considered. Numerical simulations are carried out using a (3D) MHD code developed by our research group, first at a fixed $\operatorname{Ra}=10^{5}$ and $\operatorname{Pr}=0.71$ for aspect ratios $A=$ 2, 4,6, 8 and Hartmann numbers in the range $H a=0-100$. As the aspect ratio is increased, a RayleighBénard-like convection with the convective cells formed near the symmetric central portion of the enclosure, where the mean temperature gradient is anti-parallel to the gravity, is found to be triggered. Except at the transition, the effect of the imposed radial magnetic field is found to decrease the fluid motion in general, and the convective motion is completely suppressed at $\mathrm{Ha}=100$ irrespective of the aspect ratio. The critical Hartmann number for the onset of (R-B-like) convection is found to decrease with an increase in the aspect ratio. Numerical simulations are also attempted at a fixed $A=10$ and $R a=8000$ for Prandtl numbers $\operatorname{Pr}=$ 10, 0.1, 0.01 and Hartmann numbers $H a=0,3,6,9,12$. In the absence of the applied magnetic field, the flow is found to exhibit periodic oscillations of increased amplitude and time-period when $\operatorname{Pr}$ is decreased, except at $\operatorname{Pr}=10$, where a steady-state solution is found. For $\operatorname{Pr}=0.01$, the oscillatory flow is observed to persist even when the magnetic field strength is increased in the range $H a=3-12$. Moreover, the temporal frequency of these flow oscillations is found to be nearly the same for $H a \leq 9$.
\end{abstract}

Keywords. Magnetohydrodynamic flow; natural convection; curved enclosure.

\section{Introduction}

Natural convection in the presence of an applied magnetic field has been widely studied in simple enclosure geometries [1-3]. More complex geometries such as annuli are currently being studied, wherein an intricate flow behaviour is observed. The different flow patterns that are produced in these configurations result not only from the geometry itself, but also from the action of the Lorentz force generated by the imposed magnetic field.

Joshi [4] obtained an analytical solution for the fully developed natural convection in a vertical annulus considering uniform wall temperature. A numerical investigation [5] of natural convection in a horizontal annulus confirmed that the critical Rayleigh number at which convection is initiated decreases with an increase in the annular gap, and

*For correspondence that when convection occurs, two counter-rotating cells appear in the enclosure. Mizushima et al [6] theoretically and numerically investigated the transition behaviour of the flow field for various Rayleigh number using bifurcation analysis.

An exact solution in the presence of a radial magnetic field was obtained by Singh et al [7] for vertical concentric annuli. Singh and Singh [8] numerically studied the effect of the induced magnetic field on the natural convection in the same configuration. Their study showed that the convective flow velocity is reduced by an increase in the Hartmann number - a measure of the strength of magnetic field, and represents the ratio of the square root of Lorentz force to the viscous force, while it increases with the induced magnetic field.

Recently, Ashorynejad et al [9] numerically studied the effect of a radial magnetic field on natural convection in a horizontal cylindrical annulus enclosure filled with a nano- 
fluid, and determined the effect of parameters such as the Hartmann number and the Rayleigh number on the flow field. They found that an increase in the Hartmann number decreases the average Nusselt number, but the latter increases with the Rayleigh number. Natural convection in a half annulus in the presence of a magnetic field for different angles of inclination was studied also by Sheikholeslami et al [10], who found secondary eddies at an angle of inclination $\theta=45^{\circ}$, but a single convection cell is found at inclinations $\theta=0^{\circ}$ and $90^{\circ}$.

The present study is concerned with the effect of a purely radial magnetic field on the natural convection of an electrically conducting fluid in a $2 \mathrm{D} 180^{\circ}$ cylindrical half-annular enclosure, for various Hartman numbers. The inner wall is kept at a higher temperature than the outer wall, while the end(-wall)s are insulated. In such a geometric configuration, the flow behaviour at large aspect ratios is expected to exhibit characteristics similar to that of the Rayleigh-Bénard flow - a problem that has been widely studied in the case of rectangular enclosures with and without an applied magnetic field [11-13]. Here, the introduction of the magnetic field in the case of an electrically conducting fluid delays the onset of convection [14], and the critical Rayleigh number increases with the Hartmann number. The orientation of the magnetic field also plays an important role in the convection roll formation [15] and furthermore, it is found that the number of convection rolls increase with an increase in the Hartmann number[16]. At small aspect ratios on the other hand, the flow in the two end arms of the enclosure where the mean temperature gradient is nearly perpendicular to that of gravity is expected to be similar to that of the Hadley configuration.

The present study, which includes determination of the effect of aspect ratio of the semi-circular enclosure, on the formation of the convection rolls, is mainly motivated by the geometry and problem configuration wherein, the mean temperature gradient varies continuously from being perpendicular at the two arms of the enclosure, to nearly antiparallel near the middle section, with respect to the gravity vector. This problem, where combined characteristics of the Rayleigh-Bénard and Hadley flows are expected to interact, therefore forms an important exercise in understanding more complex convection flows better in general.

\section{Problem statement}

A schematic of the curved enclosure is shown in figure 1 . In the present case, we define the aspect ratio as

$$
A=\frac{\pi R_{0}}{L}
$$

where $R_{0}=\left(R_{1}+R_{2}\right) / 2$ is the average radius of the annulus and $L=\left(R_{2}-R_{1}\right)$ is the characteristic length; $R_{2}$ and $R_{1}$ are the outer and inner radii of the enclosure, respectively. The semi-circular geometries considered for the present calculations are constructed with $L=1$.

The enclosure is heated at the inner wall $\left(R_{1}\right)$ and cooled at the outer wall $\left(R_{2}\right)$ while the other two walls are thermally insulated. All walls of the enclosure are assumed to be electrically perfectly conducting. The direction of gravity, $\mathbf{g}$, is along the negative $Y$ direction. The applied magnetic field, $B_{R}$, is purely radial but complies with the $\nabla \cdot \mathbf{B}=0$ condition and is taken to be $B_{R}=B_{0} \frac{R_{0}}{r}$, where $R_{1} \leq r<R_{2}$ and $B_{0}=1$. This radial magnetic field, because of the resultant Lorentz force, offers a maximum resistance to the induced flow in the azimuthal direction. The Rayleigh number and Hartmann number are defined as $R a=$ $g \beta \Delta T L^{3} / \nu \alpha$ and $H a=L B_{0} \sqrt{\sigma / \mu}$, respectively.

\section{Governing equations and boundary conditions}

\subsection{Governing equations}

The governing equations in non-dimensional form, neglecting the induced magnetic field, Joule heating and viscous dissipation in the energy equation, are as follows.

Continuity equation:

$$
\nabla \cdot \mathbf{u}=0
$$

Navier-Stokes equation:

$$
\frac{\partial \mathbf{u}}{\partial t}+(\mathbf{u} \cdot \nabla) \mathbf{u}=-\nabla P+\frac{1}{\sqrt{G r}} \nabla^{2} \mathbf{u}+\frac{H a^{2}}{\sqrt{G r}} \mathbf{J} \times \mathbf{B}-T \hat{Y} .
$$

Ohm's law:

$$
\mathbf{J}=(-\nabla \phi+\mathbf{u} \times \mathbf{B}) .
$$

Current continuity equation:

$$
\nabla \cdot \mathbf{J}=0
$$

Poisson equation for the electrical potential:

$$
\nabla^{2} \phi=\nabla \cdot(\mathbf{u} \times \mathbf{B}) .
$$

Temperature equation:

$$
\frac{\partial T}{\partial t}+(\mathbf{u} \cdot \nabla) T=\frac{1}{\sqrt{G r} P r} \nabla^{2} T
$$

Here, the Grashoff number, $G r=g \beta \Delta T L^{3} / v^{2}$, gives the ratio of buoyancy to viscous force, the Hartmann number, $H a \equiv L B_{0} \sqrt{\frac{\sigma}{\mu}}$, measures the strength of magnetic field and is the ratio of the square root of Lorentz force to the viscous force, and the Prandtl number, $\operatorname{Pr}=\frac{v}{\alpha}$, is the ratio of 


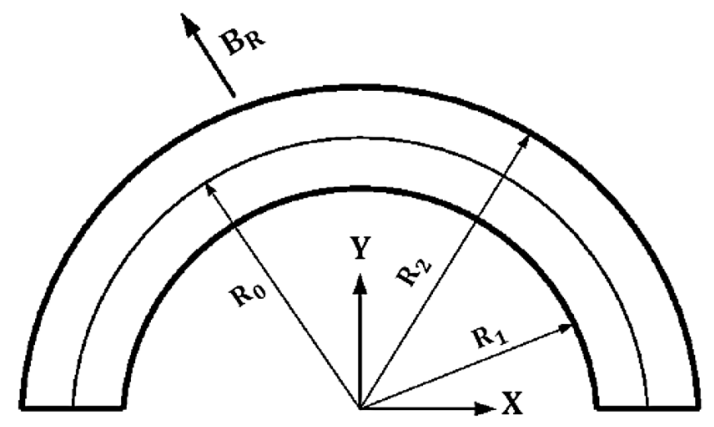

Figure 1. Schematic diagram of curved geometry.

kinematic viscosity to thermal diffusivity. The Rayleigh number is defined as the product $R a \equiv G r P r$.

To obtain these equations, the velocity field (u) is scaled by the characteristic velocity $u_{0} \equiv \sqrt{g \beta \Delta T L}$ - obtained by the balance of inertia and buoyancy force terms. Here $\Delta T \equiv T_{h}-T_{c}$, and $\beta$ is the coefficient of thermal expansion. The scale factors used for the non-dimensionalization of the other variables in the equations are

$$
\begin{aligned}
& X=x / L, Y=y / L, u=U / u_{0}, v=V / u_{0}, t \leftarrow t /\left(L / u_{0}\right) \\
& P=p / \rho u_{0}^{2} \quad \text { and } \quad T \leftarrow \frac{T-\left(T_{h}+T_{c}\right) / 2}{\left(T_{h}-T_{c}\right)} .
\end{aligned}
$$

\subsection{Boundary conditions}

The appropriate boundary conditions for the governing equations are as follows:

$$
\begin{aligned}
& u=v=\phi=0 \quad \text { and } \quad T=+0.5 \quad \text { at } \quad R_{1}, \\
& u=v=\phi=0 \quad \text { and } \quad T=-0.5 \quad \text { at } \quad R_{2}, \\
& u=v=\phi=0 \quad \text { and } \quad \frac{\partial T}{\partial n}=0 \quad \text { at } \quad Y=0 \quad \text { and } \\
& R_{1} \leq|X| \leq R_{2} .
\end{aligned}
$$

\subsection{Code validation, grid independence study and computational details}

These equations are discretized by employing the finitevolume methodology and are numerically solved using a 3D MHD code developed by our research group. The solution algorithm - a variant of the PISO, its implementation and code validation with various benchmark results are detailed in our previous paper [17] and therefore not exposited here. All the calculations performed for the present study are obtained by employing QUICK as the convection scheme and Crank-Nicolson as the temporal scheme. For its relevance to the present study, we report

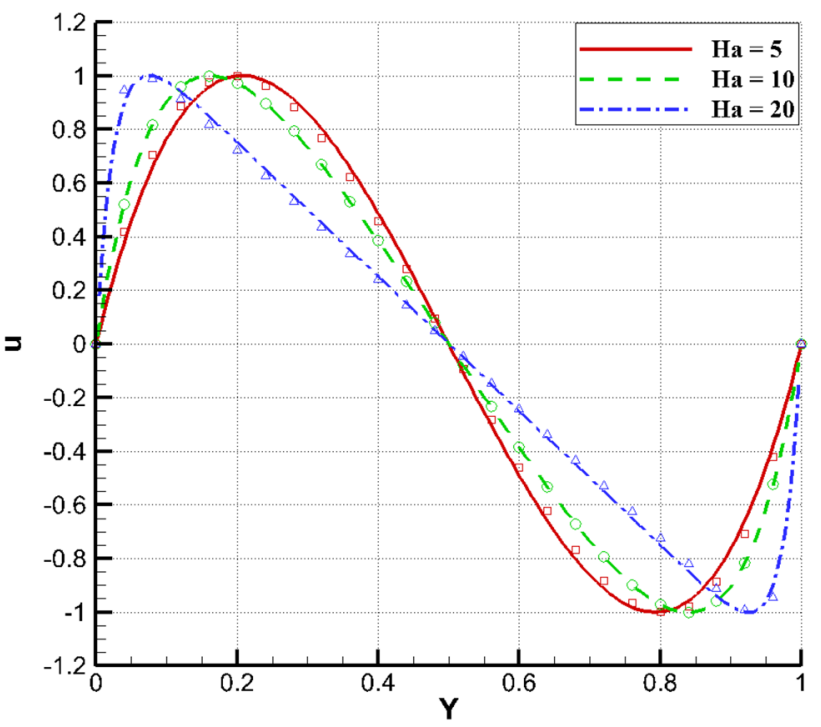

Figure 2. Comparison of normalized velocity $(u)$ with analytical solution (shown in symbols) of Garandet et al [2] as a function of centreline vertical coordinate distance at different Hartmann numbers, $H a=5-20$.

comparison of the numerical solutions obtained using our code to the analytical solutions of Garandet et al [2]. Numerical solutions are obtained for $G r=2.0 \times 10^{4}, P r=$ 0.01 and aspect ratio $A=4$. A non-uniform computational mesh of size $251 \times 101\left(N_{x} \times N_{y}\right)$ is used. The computed normalized velocity shown in figure 2 has excellent agreement with the corresponding analytical solutions at different Hartmann numbers considered.

\subsection{Grid independence study and computational details}

Grid independence tests are carried out at $\mathrm{Ha}=60, \mathrm{Pr}=$ 0.71 and $R a=10^{5}$ using a uniform grid spacing in both the radial and azimuthal directions for all aspect ratios. Figure 3 shows the test result for the case $A=8$, where the estimated error in using the mean grid $151 \times 801$ is found to be about $1.2 \%$. Similar test runs yielded $0.26 \%$ and $0.38 \%$ as the estimated errors on grids $151 \times 401$ and $151 \times 601$ for $A=4$ and $A=6$, respectively (not shown). The results presented in this paper are obtained from subsequent simulations performed on these respective grids (for each aspect ratio), where the distance of the first grid point from the wall is kept the same at $0.0066 L$.

In the present paper, we report numerical results obtained at a fixed Rayleigh number $R a=10^{5}$ with $P r=0.71$ for aspect ratios $A=2,4,6,8$ to ascertain the effect of aspect ratio. Results at various Hartman numbers in the range $H a=0$ to $H a=100$ are then presented. Lastly, results demonstrating the effect of Prandtl number on convection in the enclosure of aspect ratio $A=10$ at a fixed $R a=8000$ 


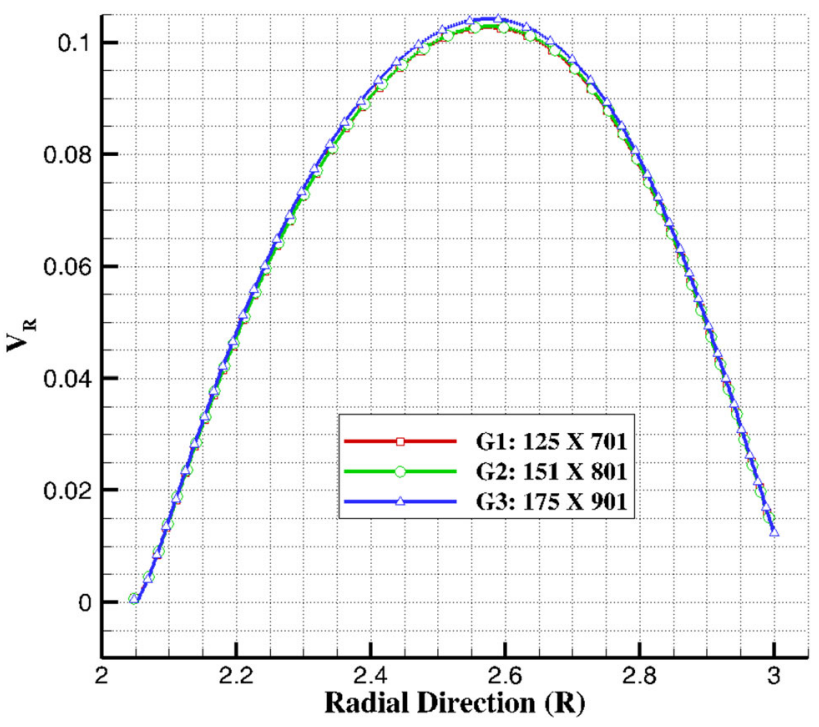

Figure 3. Grid independence test results at $A=8$.

are shown. The Prandtl numbers chosen in this case are $\operatorname{Pr}=10,0.1,0.01$ and the calculations are performed for Hartmann numbers $H a=0,3,6,9,12$. These results are discussed in detail in the next section.

\section{Results and discussion}

\subsection{Effect of aspect ratio}

In this section, we discuss the results for different aspect ratios obtained on the full domain. In the present case, we have carried out numerical simulations for $R a=10^{5}$ and $P r=0.71$. The effects of the radial magnetic field of different strengths (i.e., Hartmann number) and four different aspect ratios, $A=2,4,6,8$, are studied.

From figure 4 , it is observed that increasing the Hartmann number from $\mathrm{Ha}=0$ to 75 does not change the number of convection rolls for $A=2$, but has a pronounced effect for $A=4$, as shown in figure 5 .

As the direction of the temperature gradient $(\Delta T)$ is antiparallel to the direction of gravity $(\mathbf{g})$ at the middle section of the enclosure - a configuration analogous to the R-B system, the convective flow in that part of the enclosure is susceptible to flow instability akin to the R-B flow. This is precisely what is seen in the stream-function plots portrayed in figures 5-7 for various aspect ratios. With an increase in the aspect ratio the region in the neighbourhood of the central symmetric section of the enclosure where ( $\Delta T \| \mathbf{g}$ ) is also increased, thereby resulting in the increase in the number of R-B-like convection rolls. At the two ends of the domain, the gravity is perpendicular to the temperature gradient (similar to the Hadley convection configuration) and therefore a large circulation cell is formed at the two arms of the enclosure.
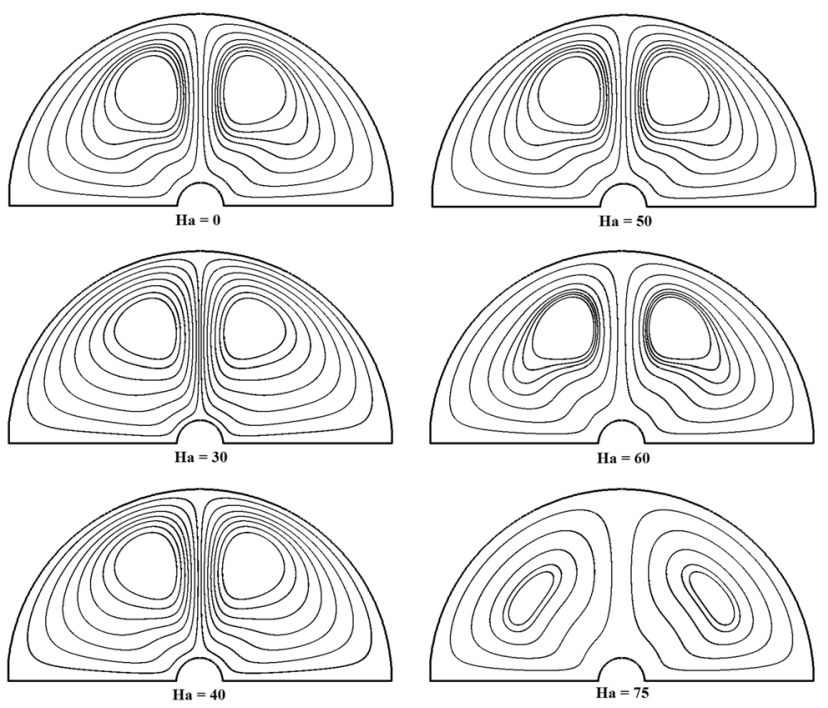

Figure 4. Streamline plots show effect of Hartmann number, $H a=0,30,40,50,60,75$, on the formation of convection rolls for aspect ratio $A=2$.

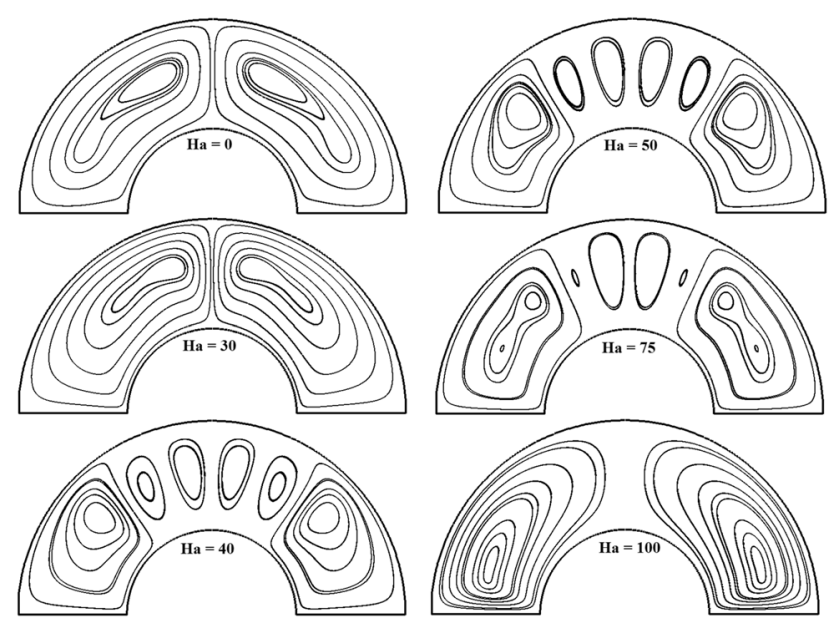

Figure 5. Streamline plots show effect of Hartmann number, $H a=0,30,40,50,75,100$, on the formation of convection rolls for aspect ratio $A=4$.

The convection rolls mainly occur due to the effect of the Lorentz force, which acts against flow in the azimuthal direction, thereby decreasing the velocity in that direction. Hence, the two large convection rolls symmetrically located on the either side of the enclosure shrink in the azimuthal direction, and the interstitial space in the middle section of the enclosure is then filled by $\mathrm{R}-\mathrm{B}$ mode convection cells if triggered.

For aspect ratio $A=2$, Hadley-like flow is dominant, and two circulation rolls form in the domain. In conventional Hadley flow we generally see a single cell but here two convection rolls are created by the curved geometry, one in each arm of the enclosure. An increase in Hartmann number up to $H a=75$ does not create more convection rolls, 

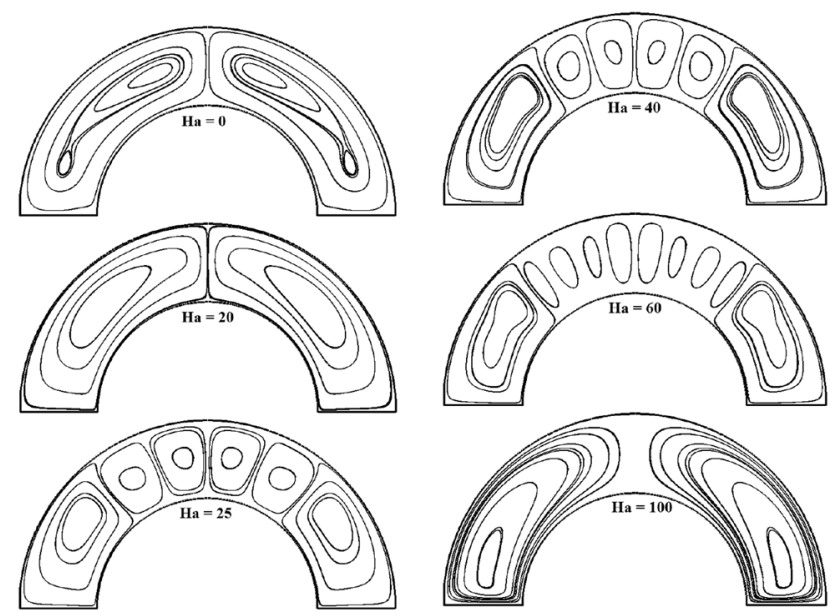

Figure 6. Streamlines plot shows effect of Hartmann number, $H a=0,20,25,40,60,100$, on the formation of convection rolls for aspect ratio $A=6$.
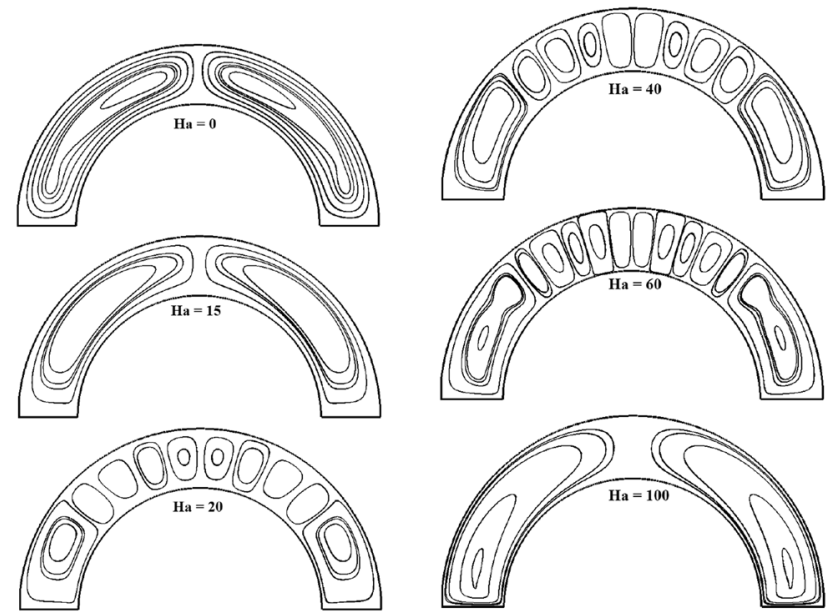

Figure 7. Streamlines plot shows effect of Hartmann number, $H a=0,15,20,40,60,100$, on the formation of convection rolls for aspect ratio $A=8$.

but the magnitude of the fluid motion is increasingly suppressed as the Hartmann number is increased.

Even for aspect ratio $A=4$ (see figure 5) rolls do not increase up to Hartmann number $H a=30$, presumably because the Hadley mode is still dominant. However, a further increase in Hartmann number from $\mathrm{Ha}=40$ to $H a=75$ increased the number of convection rolls to 6 . The convection rolls formation seems to occur also because of the action of the Lorentz force at increased magnetic field strengths, triggering a Rayleigh-Bénard-type convection mode in the middle section of the domain, which dominates over the Hadley mode. At $H a=100$ however, the fluid motion is almost completely suppressed and the flow reverts to two large, but weak, convection rolls.

A similar pattern of formation of convection rolls is observed for aspect ratios $A=6$ and 8 . Figures 6 and 7

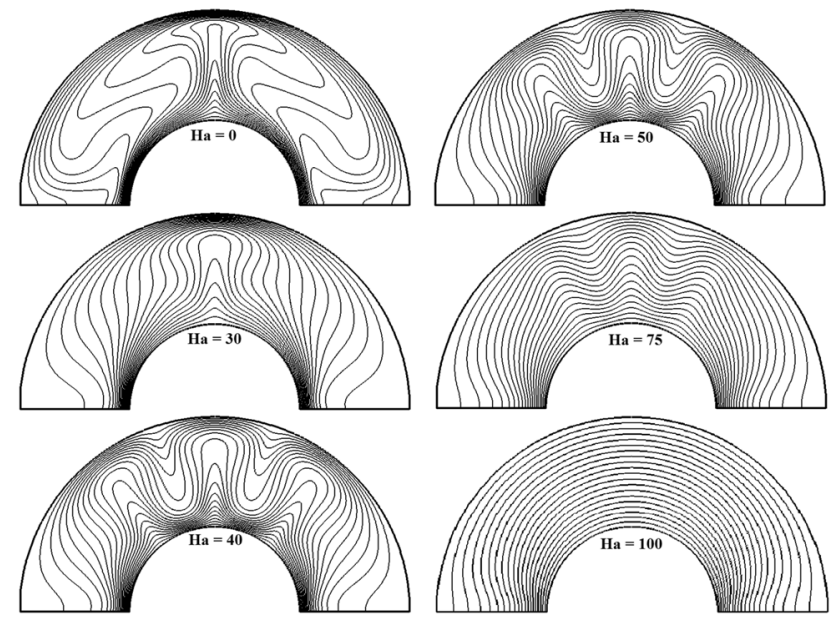

Figure 8. Temperature plots for different Hartmann numbers $H a=0-100$ at $R a=10^{5}, A=4$ and $P r=0.71$.

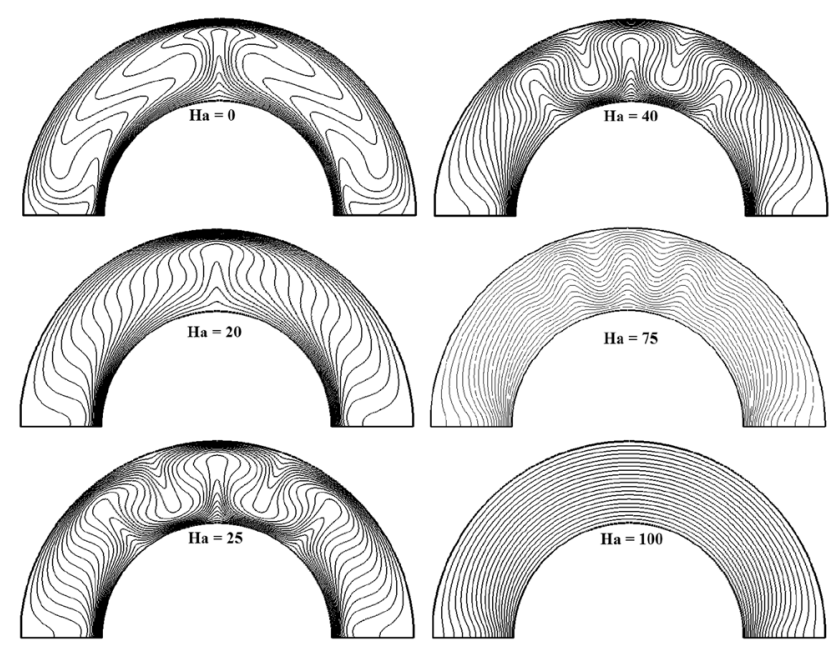

Figure 9. Temperature contour plot for aspect ratio $A=6$ at different Hartmann numbers $H a=0-100$ for $P r=0.71$ and $R a=10^{5}$.

show the streamline plots at different Hartmann numbers. The results clearly indicate that an increase in the aspect ratio increases the formation of the convection rolls. It is found that at Hartmann number of $H a=40$ and aspect ratios of $A=4, A=6$ and $A=8$, the convection rolls are found to be six, six and 10, respectively (see figures 5, 6 and 7). For all aspect ratios, we observe that the strength of velocity gets weak for $H a>75$ and at $H a=100$ the buoyancy force is not sufficient enough to form the convection rolls.

The same interpretation can also be made from the temperature contours shown in figures 8,9 and 10 for different aspect ratios $A=4,6$ and 8, respectively, wherein it is seen that the convection motion is still present at Hartmann number of $H a=75$, but is fully suppressed at $H a=$ 100 - isotherms nearly coinciding with $r=$ const. lines. 

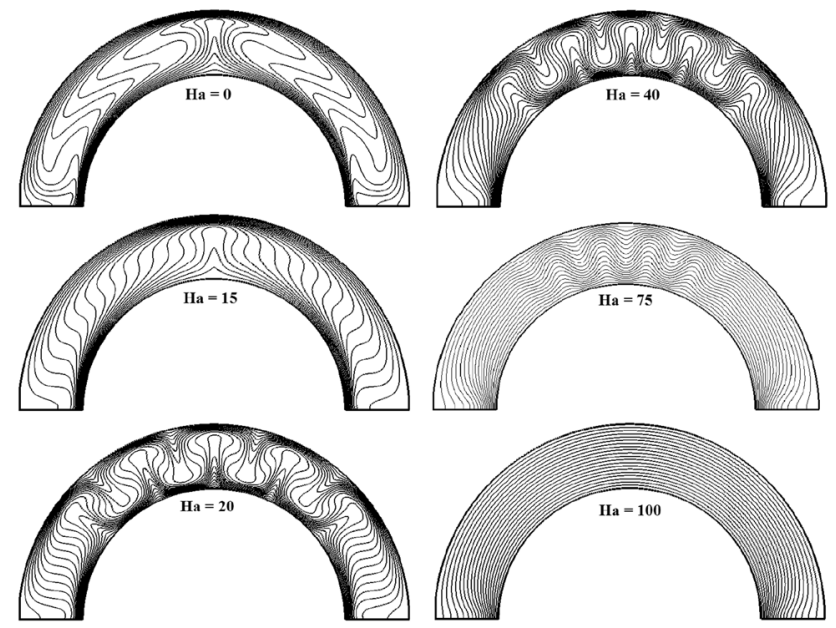

Figure 10. Temperature contour plot for aspect ratio $A=8$ at different Hartmann numbers $H a=0-100$ for $P r=0.71$ and $R a=10^{5}$.

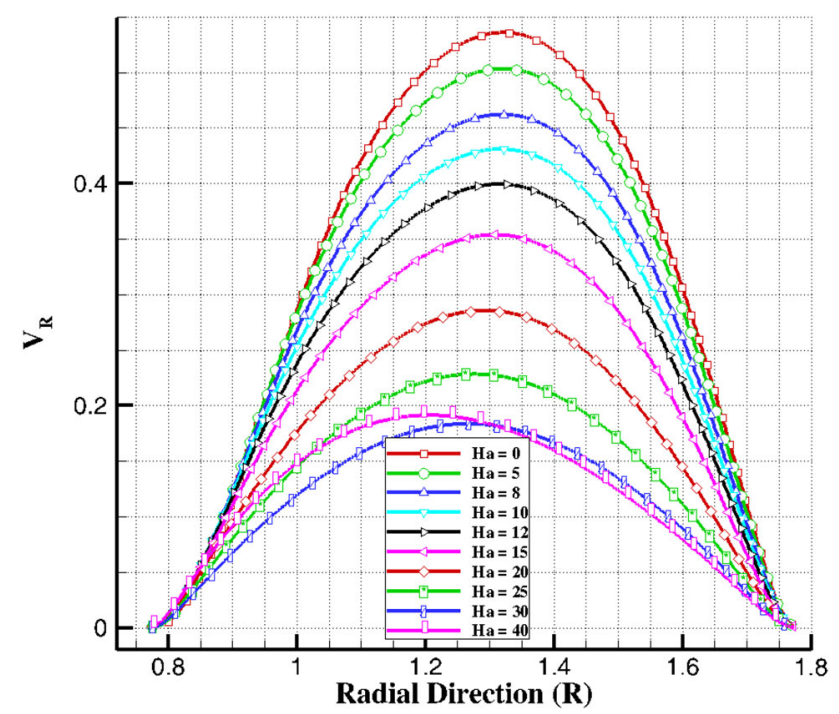

Figure 11. Effect of Hartmann number $H a=0-40$ on the velocity profiles for aspect ratio $A=4$, at $X=0$.

\subsection{Effect of Hartmann number}

In order to study the effect of Hartmann number on the fluid motion for different aspect ratios, we have chosen the Rayleigh number $R a=10^{5}$, and Prandtl number $P r=0.71$. The variation of the resultant velocity $\left(V_{R}=\sqrt{u^{2}+v^{2}}\right)$ is plotted along the radial direction, $R$, at the mid-section $(X=0)$ of enclosure as shown in figure 11. It was already seen in the earlier sections that an increase of magnetic field strength (i.e., Hartmann number) decreases the resultant velocity. We find the same pattern of velocity decrease here when the Hartmann number is increased. The same behaviour can be seen in figures 12 and 13 for $A=6$ and 8 , respectively.

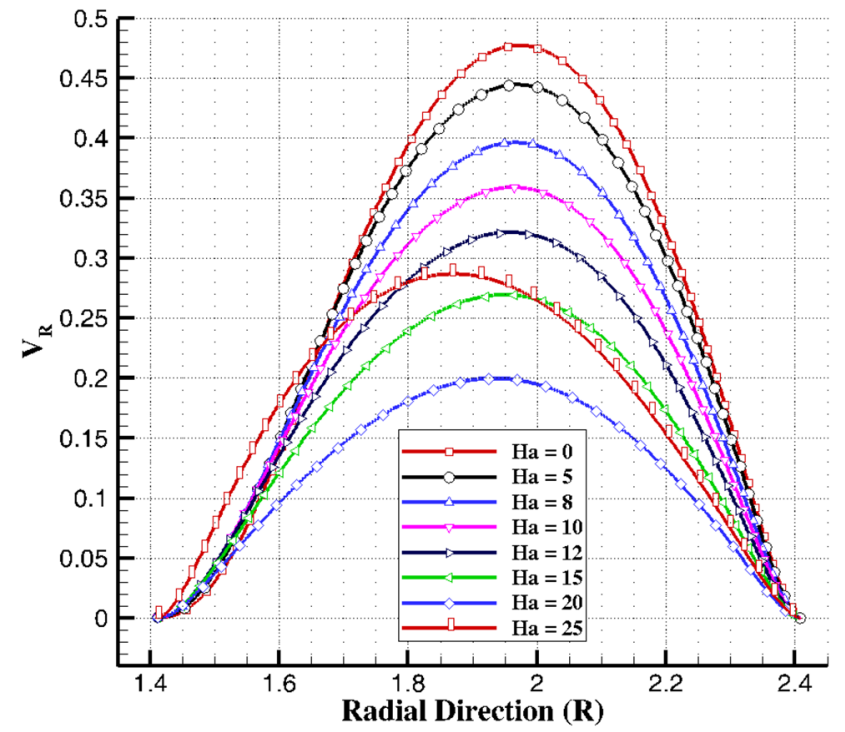

Figure 12. Effect of Hartmann number $H a=0-25$ on the velocity profiles at aspect ratio $A=6$ and $X=0$.

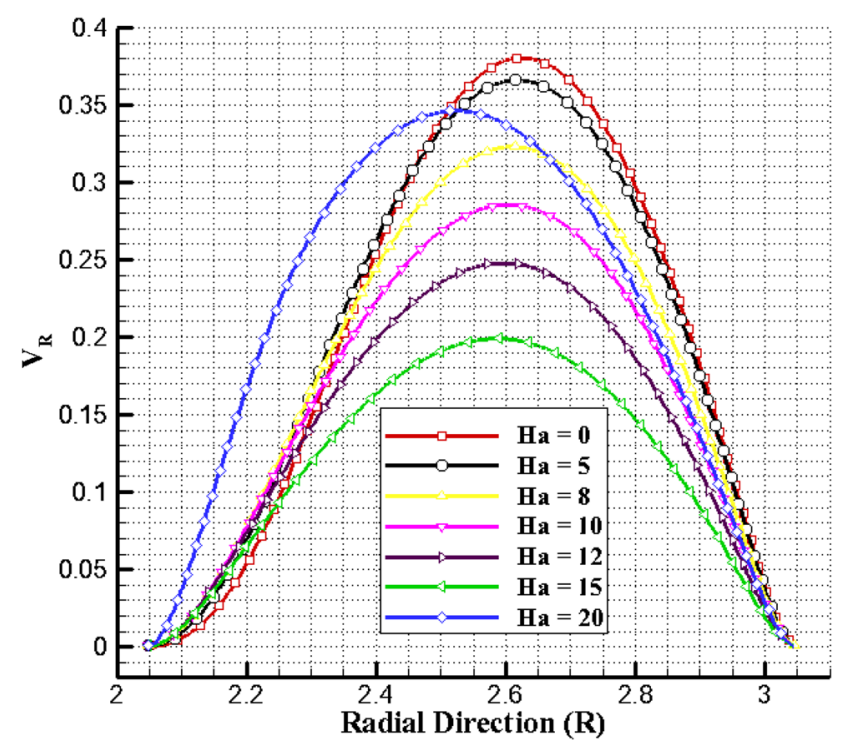

Figure 13. Effect of Hartmann number $H a=0-20$ on the velocity profiles at the mid-section of domain, $X=0$, for $A=8$.

A significant change in the velocity profile is observed at the transition point, where a circulation cell pattern breaks into more cells. We see that an increase in the Hartmann number decreases the resultant velocity both up to and beyond the transition point, but a significant increase in the resultant velocity is observed at the transition point (see figures 11-13) due to the onset of the R-B-type convection roll formation as discussed earlier.

In MHD, it is usually observed that the velocity magnitude decreases as the Hartmann number increases. The momentary velocity increase before decreasing again at a higher $\mathrm{Ha}$ as observed in the present case can then be 
Table 1. Variation of Nusselt number with various Hartmann numbers $0-100$ at $R a=10^{5}$ and $\operatorname{Pr}=0.71$ for $A=8$.

\begin{tabular}{lc}
\hline Hartmann number $\mathrm{Ha}$ & Nusselt number $N u$ \\
\hline 0 & 9.301 \\
10 & 8.167 \\
20 & 8.935 \\
30 & 6.674 \\
40 & 6.260 \\
50 & 5.110 \\
60 & 4.116 \\
75 & 2.990 \\
100 & 1.127 \\
\hline
\end{tabular}

attributed to the R-B-like mode that is triggered at the transition point and persists over a range of $\mathrm{Ha}$. This apparent breaking of the cells due to the formation of newer $\mathrm{R}-\mathrm{B}$-like convection rolls is reflected as the enhanced convection at the transition point.

The variation of Nusselt number at the hot wall with Hartmann number is shown in table 1. It is seen that an increase in the Hartmann number decreases the Nusselt number, mainly due to the increase of Lorentz force, which suppresses the fluid motion. At large $H a=100$, where the convection motion is completely suppressed, the average Nusselt number is close to 1 , indicating that the heattransfer mode is predominantly conductive. The noticeable change in the Nusselt number observed at $H a=20$, as compared with $H a=10$, is due to the formation of new convection rolls from the R-B-like mode instability as mentioned previously, which seemingly enhanced the convection motion at the transition point (not necessary at $H a=20$ but somewhere between $H a=10$ and $H a=20$.)

This transition point strongly depends on the aspect ratio. It is found that at the higher aspect ratio $A=8$ it occurs at a lower Hartmann number, $H a=20$, as compared with the lower aspect ratio $A=6$, where it occurs at $H a=25$. In the case of aspect ratio, $A=4$, an even larger value of Hartmann number $(H a=40)$ is needed for breaking of the convection roll into multiple rolls.

We have plotted the variation of square root of the critical Hartmann number with the aspect ratios in figure 14. The plot shows a linear variation of the square root of the critical Hartmann number with aspect ratios. This plot can be used to predict the quantitative behaviour of the transition point (cells break) for different aspect ratios.

\subsection{Aspect ratio $A=10$}

In this section, we report results for the very high aspect ratio case of $A=10$. The numerical computations are carried out for different Hartmann numbers $(H a=0,3,6,9,12)$, Rayleigh number $R a=8000$ and Prandtl numbers $(\operatorname{Pr}=10,0.1,0.01)$. In the first case, we

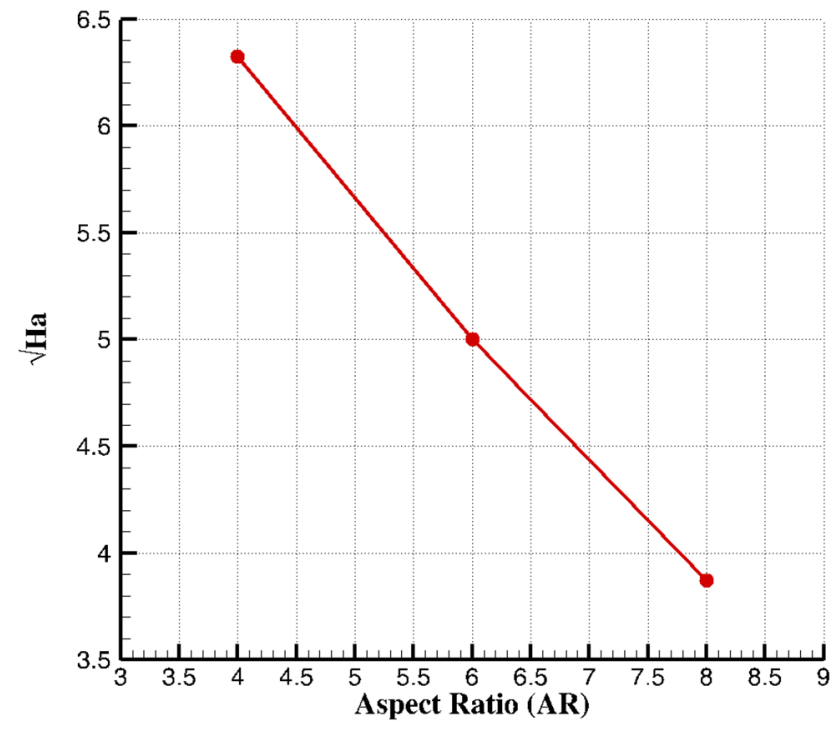

Figure 14. Variation of critical (transition) Hartmann number with aspect ratio at $R a=10^{5}$ and $\operatorname{Pr}=0.71$.

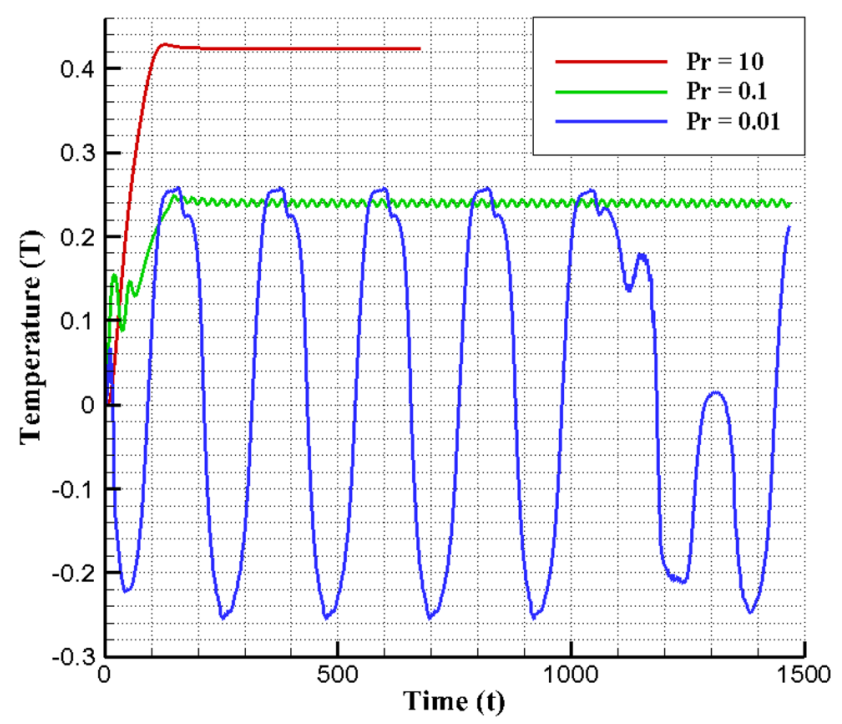

Figure 15. The temporal variation of temperature at $R a=8000$ and $H a=0$ for $\operatorname{Pr}=10,0.1,0.01$.

study the effect of decreasing Prandtl number in the range $10,0.01,0.1$, on the formation of the convection rolls at a fixed Rayleigh number $R a=8000$ for $H a=0$, i.e., in the absence of an applied magnetic field.

To study the temporal behaviour of the solutions, we monitored temperature at a fixed point at $X=0$ and $Y=3.1847$. It is seen from the temporal evolution of temperature, as portrayed in figure 15 , that a steady-state solution is reached for $P r=10$. On decreasing the Prandtl number to $\operatorname{Pr}=0.1$ the nature of solution changes to that of a periodic oscillatory flow - which is typical in low $\mathrm{Pr}$ natural convection flows. Both the amplitude and the time 

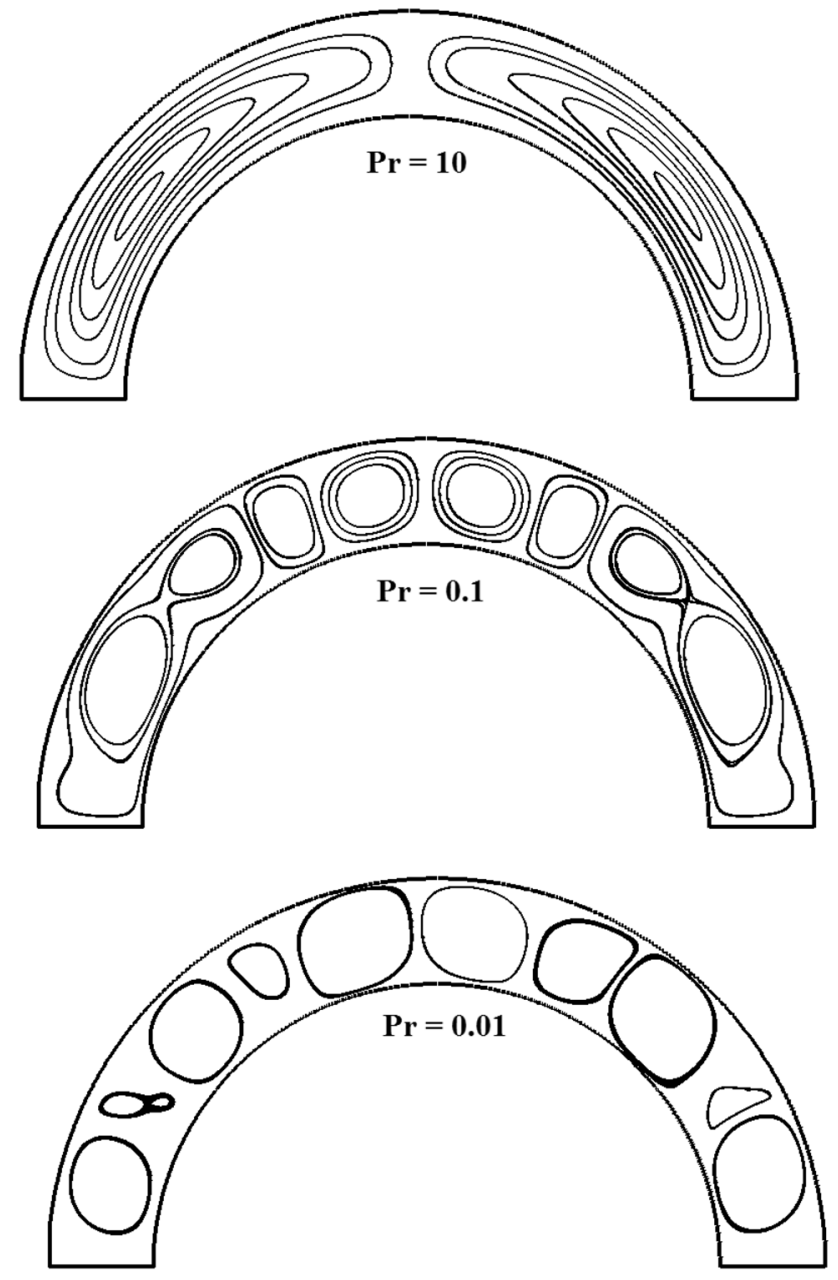

Figure 16. Streamline plots for different Prandtl numbers at $H a=0$ and $R a=8000$. Solutions for $P r=0.1$ and $P r=0.01$ are oscillatory and are shown at $t=1500$.

period of flow oscillations are increased when the Prandtl number is further lowered to $P r=0.01$. Interestingly, for $P r=0.01$, the flow began exhibiting more complex periodicity when computations were carried out over a long time beyond $t>1000$ (see figure 15), possibly due to the onset of a hydrodynamic instability. This requires a further exposition and is relegated to a future work.

The streamlines plots for different Prandtl numbers are shown in figure 16. It is seen that the Prandtl number has a significant effect on the rolls formation. At the Prandtl number of $\operatorname{Pr}=10$, we have a steady-state solution wherein a single circulation cell is observed in each half of the enclosure. As the Prandtl number is decreased, the oscillatory flow is found to be symmetric at $P r=0.1$, but asymmetric at $\operatorname{Pr}=0.01$.

For $\operatorname{Pr}=0.01$, even when the magnetic field is present, we see oscillatory flow solutions for $H a=3,6,9,12$, as shown in figure 17. Unlike the case of $H a=0$, where a different temporal evolution pattern is seen beyond

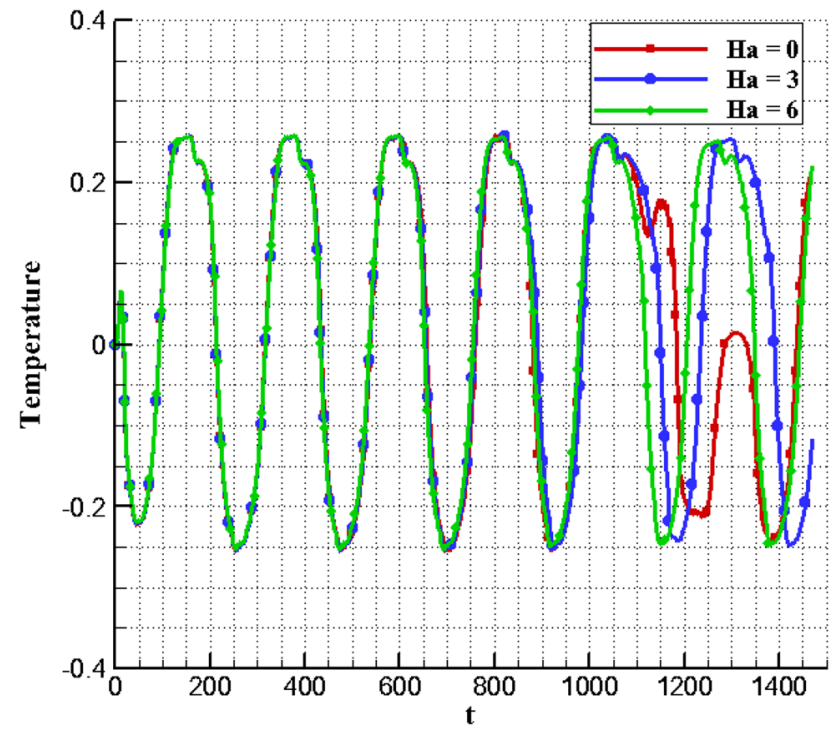

(a)

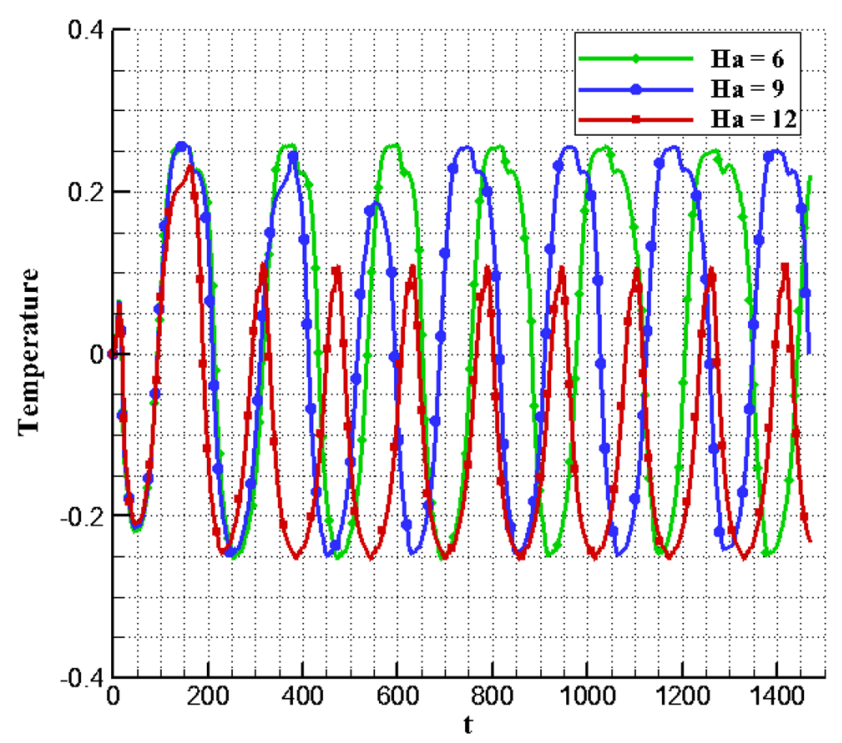

(b)

Figure 17. The temporal variation of temperature at $R a=8000$ and $P r=0.01$ for (a) $H a=0,3,6$ and (b) $H a=6,9,12$.

$t=1000$, the oscillatory flow solutions at the other Hartmann numbers are unaltered.

The characteristic frequency of flow oscillations is found to be strongly dependent on $\operatorname{Pr}$ but relatively insensitive to the Hartmann number. For $P r=0.01$, this frequency is found to be about $f \approx 0.0045$ (in the inverse scale of the time period $\tau$ ) for $H a<12$. At $H a=12$ however, the frequency is found to be around $f \approx 0.006$. The increase in the frequency might be related to the reduced cell length (and hence the wave-number of the instability), when the magnetic field strength is increased. A decrease in the amplitude of oscillations is also noticed as the Hartmann number is increased from $H a=9$ to $H a=12$. 


\section{Conclusion}

A parametric study concerning the effect of the Hartmann number, aspect ratio and Prandtl number was conducted in the case of natural convection in a semi-circular enclosure. At moderate Hartmann numbers, a Rayleigh-Bénard-type flow instability resulting in multiple convective rolls is found to occur in the middle symmetric portion of the enclosure as the aspect ratio is increased. The critical Hartmann number required to split these convection rolls is strongly dependent on the aspect ratio, with the transition Hartmann number decreasing with an increase in the aspect ratio beyond $A=2$. Except at the point of transition, the fluid motion, in general, is found to decrease and is completely suppressed at $H a=100$. Simulations performed at a fixed $A=10$ and $R a=8000$ show a change in the nature of flow solutions from steady state to periodic oscillations as the Prandtl number is decreased. Moreover, in the range of Hartmann numbers considered, the time period of these oscillations is found to be dependent strongly on the $\mathrm{Pr}$, but is relatively insensitive to an increase in the Hartmann number. At $P r=0.01$, the observed flow pattern remains periodic, but both the amplitude and frequency are modified as the Hartmann number is increased from $H a=9$ to $H a=12$.

\section{Nomenclature}

$\begin{array}{ll}R_{1} & \text { inner radius of enclosure } \\ R_{2} & \text { outer radius of enclosure } \\ R_{0} & \text { average radius of annulus }\left(R_{0}=\left(R_{1}+R_{2}\right) / 2\right) \\ L & \text { characteristic length }\left(L=R_{2}-R_{1}\right) \\ \mathbf{u} & \text { non-dimensional velocity vector } \\ \mathbf{B} & \text { non-dimensional magnetic field vector } \\ \mathbf{J} & \text { non-dimensional current density vector } \\ \mathbf{E} & \text { electric field vector } \\ \phi & \text { electrical potential } \\ \mathbf{g} & \text { gravity vector } \\ B_{0} & \text { imposed magnetic field } \\ T & \text { non-dimensional temperature }\left(\frac{T-\left(T_{h}+T_{c}\right) / 2}{T_{h}-T_{c}}\right) \\ \sigma & \text { electrical conductivity of fluid } \\ \mu & \text { viscosity of conducting fluid } \\ k & \text { thermal conductivity } \\ \rho & \text { density of fluid } \\ v & \text { kinematic viscosity } \\ \alpha & \text { thermal diffusivity } \\ \beta & \text { coefficient of thermal expansion } \\ X, Y, Z & \text { non-dimensional coordinates } \\ V_{X}, V_{Y}, V_{Z} & \text { components of the velocity field } \\ V_{R} & \text { resultant velocity } \\ \tau & \text { time-period of oscillation } \\ T_{h} & \text { hot wall } \\ T_{c} & \text { cold wall } \\ H a & \text { Hartmann number }\left(=L B_{0} \sqrt{\sigma / \mu}\right) \\ P r & \text { Prandtl number }(=v / \alpha) \\ G r & \text { Grashof number }\left(=g \beta \Delta T L^{3} / v^{2}\right) \\ & \end{array}$

$\begin{array}{ll}R a & \text { Rayleigh number }(=G r P r) \\ N u & \text { Nusselt number } \\ R_{m} & \text { magnetic Reynolds number }\end{array}$

\section{References}

[1] Fumizawa M 1980 Natural convection experiment with liquid $\mathrm{NaK}$ under transverse magnetic field. Journal of Nuclear Science and Technology 17: 98-105

[2] Garandet J, Alboussiere T and Moreau R 1992 Buoyancy driven convection in a rectangular enclosure with a transverse magnetic field. International Journal of Heat and Mass Transfer 35: 741-748

[3] Rudraiah N, Barron R, Venkatachalappa M and Subbaraya C 1995 Effect of a magnetic field on free convection in a rectangular enclosure. International Journal of Engineering Science 33: 1075-1084

[4] Joshi H 1987 Fully developed natural convection in an isothermal vertical annular duct. International Communications in Heat and Mass Transfer 14: 657-664

[5] Yoo J S 1996 Dual steady solutions in natural convection between horizontal concentric cylinders. International Journal Heat and Fluid Flow 17: 587-593

[6] Mizushima J, Hayashi S and Adachi T 2001 Transitions of natural convection in a horizontal annulus. International Journal Heat and Mass Transfer 44: 1249-1257

[7] Singh S, Jha B and Singh A 1997 Natural convection in vertical concentric annuli under a radial magnetic field. Heat and Mass Transfer 32: 399-401

[8] Singh R and Singh A 2012 Effect of induced magnetic field on natural convection in vertical concentric annuli. Acta Mechanica Sinica 28: 315-323

[9] Ashorynejad H R, Mohamad A A and Sheikholeslami M 2013 Magnetic field effects on natural convection flow of a nanofluid in a horizontal cylindrical annulus using Lattice Boltzmann method. International Journal of Thermal Sciences 64: 240-250

[10] Sheikholeslami M, Gorji-Bandpy M, Ganji D and Soleimani S 2013 Effect of a magnetic field on natural convection in an inclined half-annulus enclosure filled with $\mathrm{Cu}$-water nanofluid using CVFEM. Advanced Powder Technology 24: 980-991

[11] Clever R and Busse F 1981 Low-Prandtl-number convection in a layer heated from below. Journal of Fluid Mechanics 102: 61-74

[12] Corcione M 2003 Effects of the thermal boundary conditions at the sidewalls upon natural convection in rectangular enclosures heated from below and cooled from above. International Journal of Thermal Sciences 42: 199-208

[13] Zierep J 2003 Rayleigh-Bénard convection with magnetic field. Theoretical and Applied Mechanics 30: 29-40

[14] Chandrasekhar S 1952 XLVI. On the inhibition of convection by a magnetic field. The London, Edinburgh, and Dublin Philosophical Magazine and Journal of Science 43: 501-532 
[15] Alchaar S, Vasseur P and Bilgen E 1995 The effect of a magnetic field on natural convection in a shallow cavity heated from below. Chemical Engineering Communications 134: 195-209

[16] Möiner R and Müller U 1999 A numerical investigation of three-dimensional magnetoconvection in rectangular cavities. International Journal of Heat and Mass Transfer 42: 1111-1121

[17] Gajbhiye N, Praveen T and Eswaran V 2018 Validation and verification of a robust 3-D MHD code. Journal of Fusion Engineering and Design 128: 7-22 